\title{
DETERMINACIÓN DE FACTORES ARO ASOCIADO A MUERTES MATERNAS CON DATA MINING EN EL HOSPITAL REGIONAL DOCENTE MATERNO INFANTIL - HUANCAYO
}

\section{ARO FACTORS DETERMINING ASSOCIATED WITH MATERNAL DEATHS DATA MINING IN MATERNAL CHILD REGIONAL HOSPITAL- HUANCAYO}

\author{
Henry George Maquera Quispe, Richard Yuri Mercado Rivas, José Luis Cerrón Pérez \\ Instituto de Investigación de la Facultad de Ingeniería de Sistemas
}

\begin{abstract}
RESUMEN
El presente trabajo de investigación ha sido desarrollado debido a la importancia que tiene hoy en día la protección de las madres puérperas, ya que en los últimos años se han venido produciendo un incremento en las muertes maternas en nuestra región. Debemos manifestar que las muertes maternas hoy en día son consideradas factores de subdesarrollo. El Hospital Regional Docente Materno Infantil cuenta en la actualidad con un Sistema de Información denominado SIP-2000 (Sistema Informático Perinatal - 2000), desarrollado por el Ministerio de Salud en colaboración con organismos internacionales como por ejemplo USAID. El sistema SIP-2000 permite el registro de diferentes datos que pertenecen a las puérperas que acuden al centro de salud desde sus controles prenatales, del proceso de parto y de postparto. Esta información que se registra considera múltiples factores como los de alto riesgo obstétrico (ARO), todos ellos relacionados con la puérpera como por ejemplo: abortos, cesáreas, edad, procedencia, número de partos previos, morbilidades venéreas, etc. Todos estos datos pueden ser procesados y analizados para poder determinar los factores que son motivo de muerte en las puérperas. Utilizando los modelos de análisis de datos que proporciona la minería de datos; la investigación proporcionará como resultado las variables directas del alto riesgo obstétrico que influyen en la muerte materna. Factores que deben ser tomados en cuenta por los niveles de gobierno respectivo a fin de cambiar la situación tan agobiante que vive nuestra región central.
\end{abstract}

Palabras clave: minería de datos, riesgo obstétrico, muerte materna

\begin{abstract}
This research work has been developed because of the importance of today protecting postpartum mothers, since in recent years have been producing an increase in maternal deaths in our region. We must show that maternal deaths today are considered underdevelopment factors. The Maternal Child Regional Hospital now has an information system called SIP-2000 (Perinatal Information System - 2000), developed by the Ministry of Health in collaboration with international agencies such as USAID. The SIP-2000 system allows registration of various data pertaining to postpartum women who come to the health center from their prenatal, birth process and postpartum. This information is recorded considers multiple factors such as the high-risk obstetrics (ARO), all related to the puerperal like: Abortions, C-Section, age, origin, number of previous births, venereal morbidities, etc. All these data can be processed and analyzed to determine the factors that are a cause of death in postpartum women. Using models that provides data analysis data mining, research results provide direct variables influencing high-risk obstetrics in maternal death. Factors to be taken into account by the respective levels of government in order to change the so oppressive situation our mid region lives.
\end{abstract}

Key words: Data Mining, Danger Obstetrics, Maternal Death 


\section{INTRODUCCIÓN}

El presente trabajo de investigación está enfocado a establecer las pautas en las diferentes instancias del Ministerio de Salud, la Dirección Regional de Salud Junín, las redes de salud, micro redes de salud, y los establecimientos de salud a nivel regional para disminuir la mortalidad materna y perinatal. Del mismo modo reducir las complicaciones del embarazo, parto y puerperio de las mujeres que acuden a una atención obstétrica en el hospital regional docente materno infantil de la ciudad de Huancayo. Para lograr este objetivo nos remitimos a un sistema de información, en el que se registran todos los detalles referidos al control prenatal, atención del parto, del egreso materno y del recién nacido, además de las patologías de los recién nacidos y de las gestantes, procesamos la información y analizamos todos los registros utilizando herramientas avanzadas de minería de datos con algoritmos de árboles de decisión. Luego de los cuales logramos asegurar un sistema de información de calidad, que identificó los factores que se relacionan con las muertes maternas y que servirá para la toma de decisiones en la reducción de la mortalidad materna perinatal, en todos los sectores y niveles de gobierno.

\section{MATERIAL Y MÉTODOS}

La investigación se desarrolló utilizando como base y fuente de información el sistema informático materno perinatal SIP2000, que el Ministerio de Salud ha implementado en todas las dependencias prestadoras de salud. EI SIP2000 es un programa computacional en ambiente Windows, que permite registrar los datos de las gestantes que acuden a un establecimiento de salud, monitoriza y evalúa la evolución en forma individual y agregada, a través de reportes de gestión y seguimiento, así como indicadores construidos para diferentes estados de la gestación: control prenatal, parto, aborto, recién nacido, egreso materno y del recién nacido; en apoyo a la toma de decisiones basados en evidencia en los servicios materno perinatales. Con las herramientas de Inteligencia de Negocios (BI), como por ejemplo: el sistemas de integración de datos (SSIS), el sistemas administradores de bases de datos (MDB), el sistema de análisis de datos (SSAS); el grupo investigador analizó la información histórica de los miles de registros procesados desde el año 1999 hasta la fecha en el sistema de información Perinatal SIP-2000 y a través del algoritmo de árboles de decisión se generó un modelo de minería de datos mediante la creación de una serie de divisiones (denominadas nodos) en el árbol. El algoritmo agrega un nodo al modelo cada vez que un campo de entrada está correlacionado de forma significativa con el campo de predicción.

\section{RESULTADOS}

Mediante la herramienta SQLServer2008 y el complemento de minería de datos para Microsoft Office 2007 se logró obtener un diagrama de árbol de decisiones en el cual se identifican las múltiples variables que intervienen en la variable bajo análisis es decir las que están estrechamente relacionadas con las muertes maternas.

En el primer bloque del árbol que se muestra a continuación se puede analizar las variables iniciales que se relacionan con la evaluada en este caso la variable var092 que es la que registra las muertes maternas.

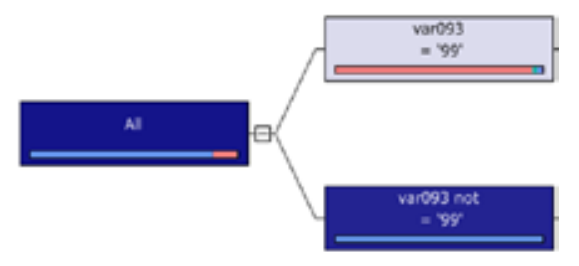

La variable var093 que según el diccionario de datos es anticoncepción es uno de los factores que influye de manera directa en los factores que son causa de muerte materna.

A continuación evaluaremos los que estén estrechamente relacionados con la anticoncepción, es decir la variable var093.

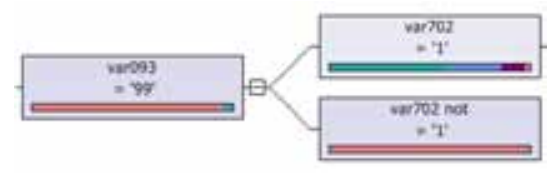


Determinación de factores aro asociado a muertes maternas con Data Mining en el Hospital Regional Docente Materno Infantil - Huancayo
Henry George Maquera Quispe, Richard Yuri Mercado Rivas, José Luis Cerrón Pérez
Esta variable es influenciada por la variable var702 que según el diccionario de datos hace referencia al Reingreso materno que generalmente ocurre luego de haberse atendido el parto y que se da por motivos de malas prácticas durante la atención a la puérpera.

A continuación analizamos las dependencias de la variable var702 de Reingreso materno.

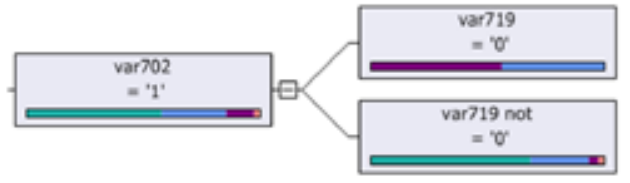

De acuerdo al grafico anterior se visualiza que este reingreso es porque la paciente tuvo un control puerperal posterior al parto que es la definición de la Variable var719 según el diccionario de datos y que fue motivo para un reingreso del paciente.

Otra variable sujeto de análisis es la variable var093 en su valor de negado, visualicemos el siguiente gráfico.

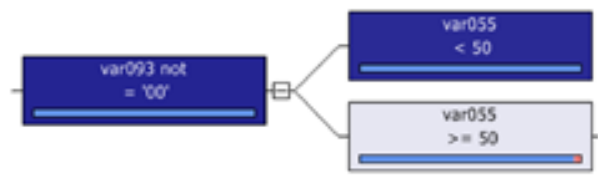

Como se puede ver las relaciones a esta condición de la variable var093 son los valores de menores de 50 y mayores o iguales que 50 para la definición como Edad gestacional.

Finalmente analizaremos las dependencias de la variable var055.

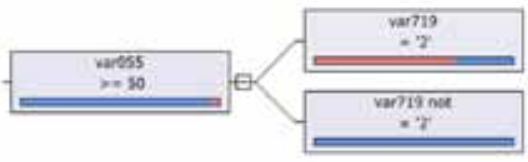

Como podemos apreciar la variable var055 también se relaciona con la variable var719 de Control Puerperal.

\section{DISCUSIÓN}

Luego de todo el proceso de análisis de las interrelaciones que tienen las diferentes variables inidentificadas por la herramienta de minería de datos y en particular por el algoritmo de árboles de decisión podemos afirmar que los factores que influyen en las muertes maternas son a causa de los reingresos maternos y la edad gestacional de las puérperas. Variables var055 y var702 del diccionario de datos del sistema materno perinatal SIP2000.

\section{CONCLUSIONES}

- Las causas de muerte maternal identificadas con la herramienta de minería de datos de SQL Server y Microsoft Office 2007 son los reingresos maternos y la edad gestacional.

- La intervención en el sistema de salud y en particular al proceso de difusión de los controles previos y posterior a la atención de la puérpera; permitirá reducir el índice de mortalidad materna en la región central ya que a través de estos controles se realizan los reingresos maternos.

- Del mismo modo la promoción de los servicios de salud relacionados con informar a la población las condiciones ideales de embarazo; como por ejemplo el factor edad gestacional permitirá reducir también el índice de mortalidad materna.

\section{REFERENCIAS BIBLIOGRÁFICAS}

1. Campbell 0 , Graham J. Strategies for reducing maternal mortality: getting on with what works. The lancet 2006.

2. Carbone C. F., Del estigma de la mortalidad materna, a la maternidad segura y saludable. Lima, 2005

3. DGE-MINSA. Análisis de la situación del Perú 2005. Serie Análisis de la situación de salud. Lima, 2006.

4. MINSA. Avanzando hacia una maternidad segura en el Perú: derecho de todas las mujeres. Lima, 2006. 
5. MINSA. Documento técnico: Estándares e indicadores de calidad en la atención materna y perinatal en los establecimientos que cumplen con funciones obstétricas y neonatales. Lima, 2007.

6. Ortiz, M., Mhoy, Mortalidad materna en el siglo XXI, Agenda Salud 28: ¿De qué mueren las mujeres?

\section{Isis Internacional.}

8. OMS. Nuevo modelo de control prenatal de la OMS. Ginebra, 2003.

9. Han, J., y Kamber, M. Data mining: concepts and techniques. USA, Academic Press. 2001

10. Witten, I. y Frank, E. Data mining: practical machine, learning tools and techniques with Java implementations. USA, Morgan Kauffmann Publishers. 1999 